
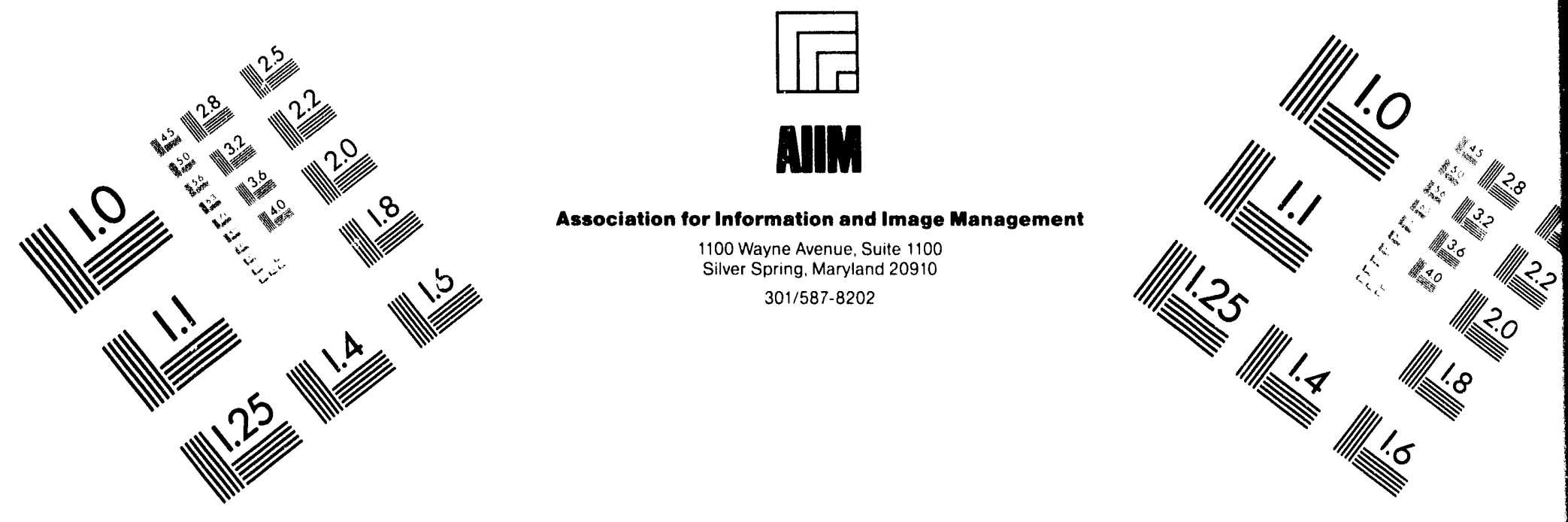

\title{
Centimeter
}

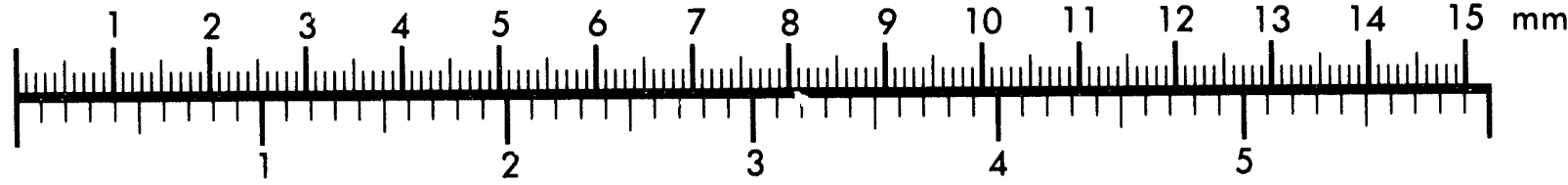
Inches
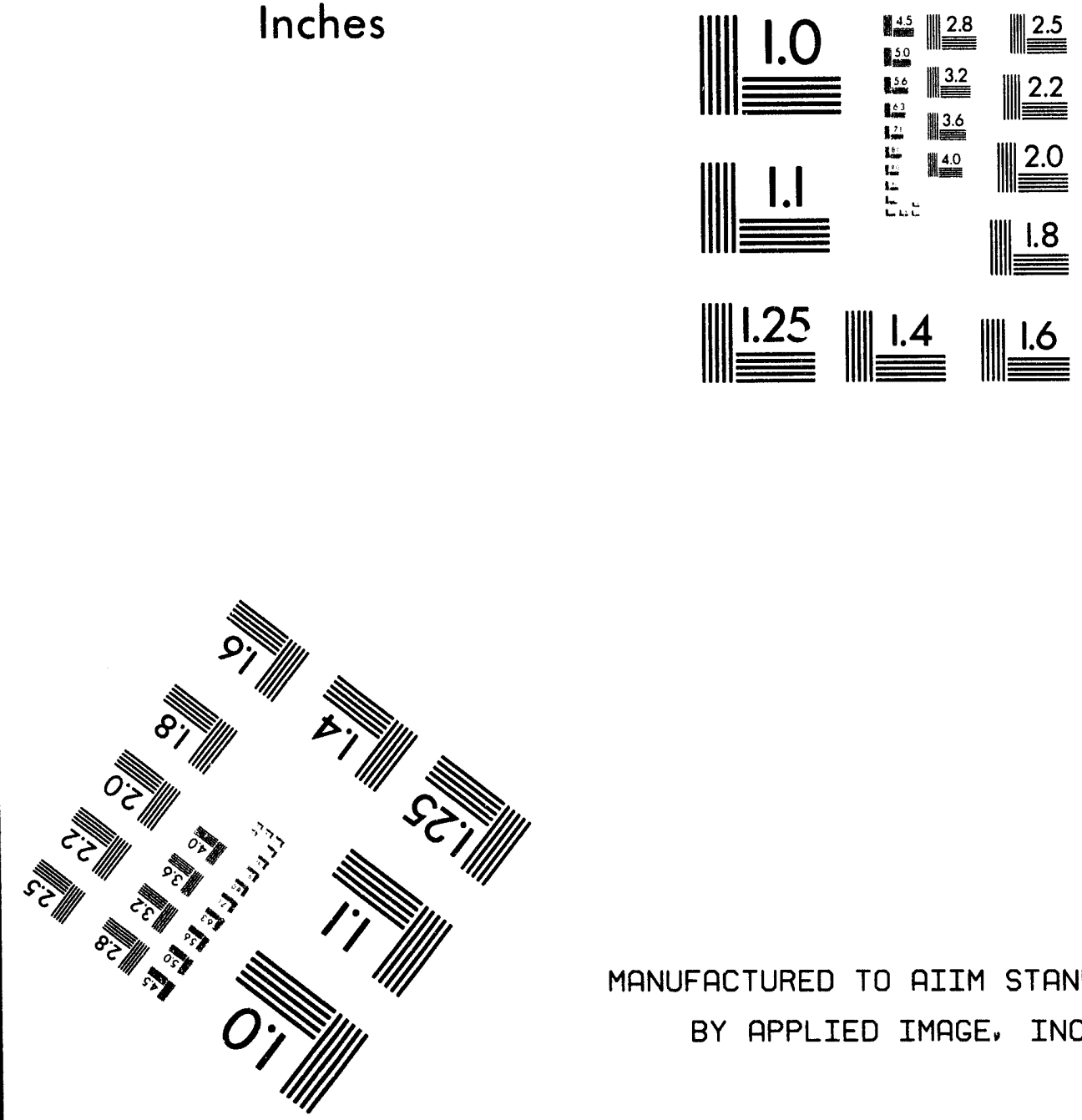

MANUFACTURED TO AIIM STANDARDS BY APPLIED IMAGE, INC.

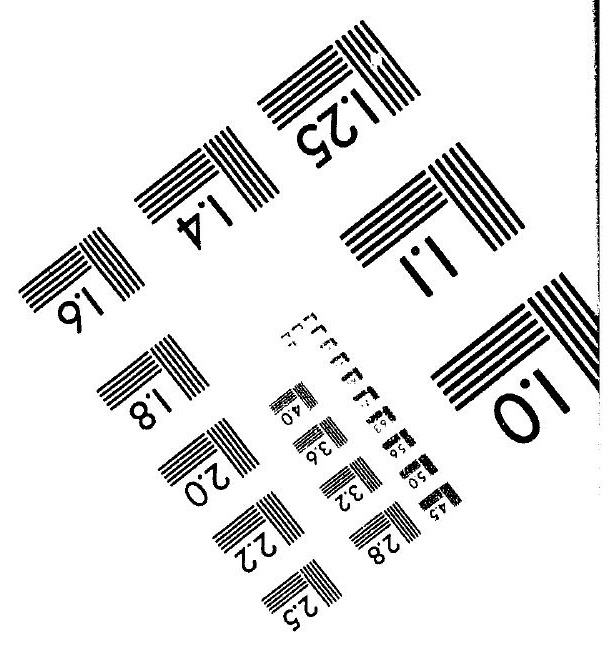



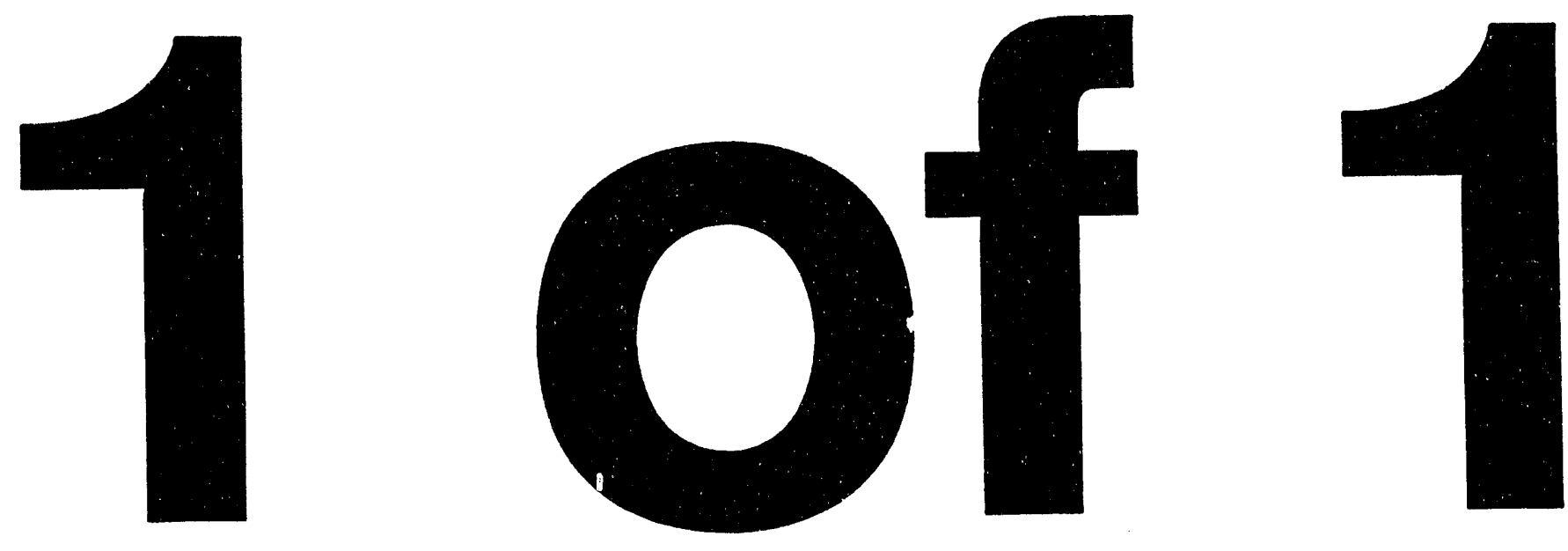


\section{LA-UR- $94-2400$}

CHROMIUM IN AQUEOUS NITRATE PROCESS STREAMS: CORROSION OF 316 STAINLESS STEEL AND CHROMIUM SPECIATION

Author(s):

Wayne H. Smith Geraldine Purdy

\section{DISCLAIMER}

Submitted to:

This report was prepared as an account of work sponsored by an agency of the United States Government. Neither the United States Government nor any agency thereof, nor any of their employees, makes any warranty, express or implied, or assumes any legal liability or responsibility for the accuracy, completeness, or usefulness of any information, apparatus, product, or process disclosed, or represents that its use would not infringe privately owned rights. Reference herein to any specific commercial product, process, or service by trade name, trademark, manufacturer, or otherwise does not necessarily constitute or imply its endorsement, recommendation, or favoring by the United States Government or any agency thereof. The views and opinions of authors expressed herein do not necessarily state or reflect those of the United States Government or any agency thereof.

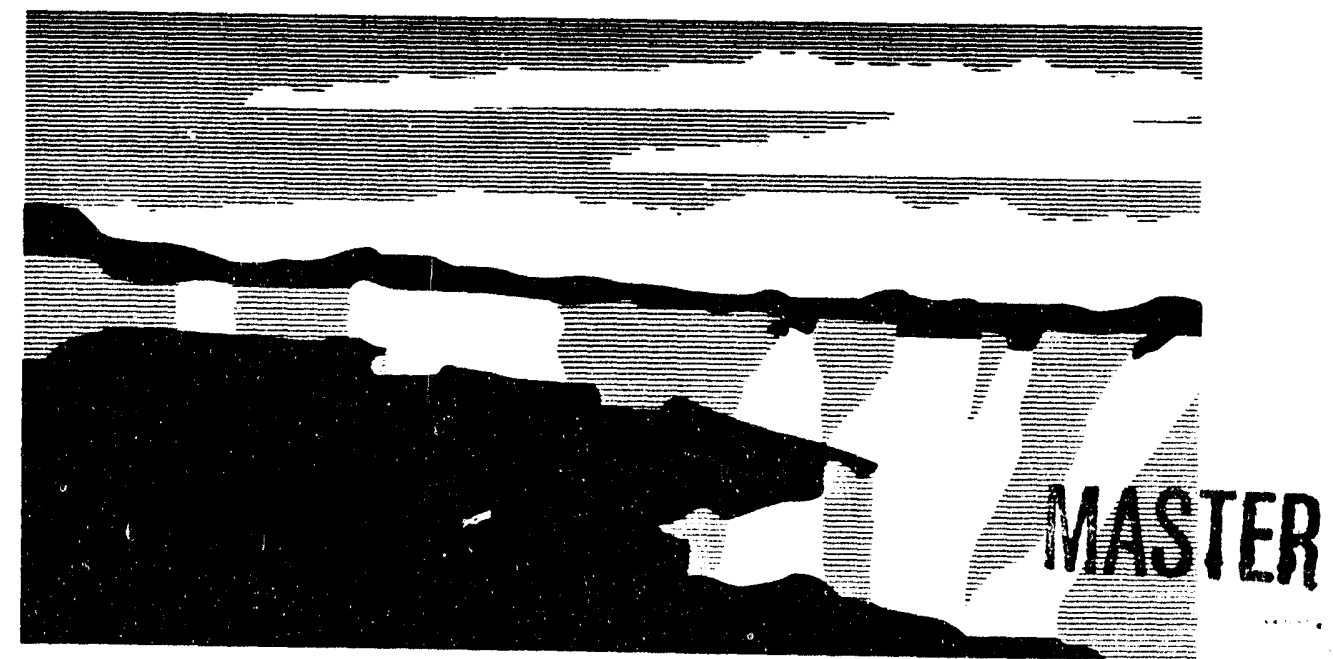

Los Alamos National Laboratory, an aff rmative action/equal opportunity employer, is operated by the University of California for the U.S. Department of Energy under contract W-7405-ENG-36. By acceptance of this article, the publisher recognizes that the U.S. Government retains a nonexclusive, royalty-free license to pubs or reproduce the published forr of this contribution, or to allow others to do so, for U.S. Government purposes. The Los Alamos National Laboratory 
Chromium in Aqueous Nitrate Plutonium Process Streams: Corrosion of 316 Stainless Steel and Chromium Speciation.

by

Wayne H. Smith and Geraldine Purdy

I. INTRODUCTION

The majority of plutonium recovery and purification operations are carried out in aqueous nitric acid solution. The waste streams generated by these operations typically contain very low levels of actinide elements as well as significant quantities of alkali, alkaline earth, and heavy metal salts. The final step in the process is to pass the waste solutions through an evaporator to distill off and recycle the nitric acid while simultaneously concentrating and collecting the salts. The salts are then fixed in cement and sent for ultimate disposal.

Two problems exist with salt disposal. First, if one of the heavy metals in the salt cake is classified as a toxic metal, then the waste designation can be elevated from lowlevel rad waste to mixed hazardous waste, depending on its concentration. This classification impacts tremendously on 
the manner and cost of disposal. Second, studies on the leaching of selected heavy metals from various types of concrete show some toxic metals are leachable. This finding again has an impact on the manner and cost involved with long-term storage.

Chromium is one of those elements that fall into these two problem areas. It is known to leach out of gypsum-type cement, the kind presently used for salt disposal, and it is listed as a toxic metal at concentrations greater than 5 ppm. However, chromium exhibits two stable oxidation states in aqueous acidic media, +6 and +3 . The +6 state is considered toxic while the +3 state is benign. Therefore, it is of interest to know not only the total chromium concentration in the waste solution but also its oxidation state.

The major source of chromium in the process streams is from the dissolution of the 316 stainless steel components ubiquitous to plutonium recovery lines. These iron alloys contain from $16 \%$ to $19 \%$ chromium and $8 \%$ to $14 \%$ nickel along with other elements in lower concentrations. These materials are relatively inert but do slowly dissolve under the combined action of nitric and hydrofluoric acids, two standard constituents of plutonium process streams. The first component of this work was to study the rate of corrosion of stainless steel in these acid solutions. 
The first stable oxidation state for chromium on dissolution of stainless steel is the +3 state. Further oxidation to the +6 state would require a very powerful oxidizing agent. The only reagent typically found in process solutions capable of carrying out this reaction is hot, concentrated nitric acid. A second component of this work was to investigate this reaction.

Once in the +6 oxidation state, chromium is itself a powerful oxidizing agent, which undergoes reduction back to chromium(+3) during the course of a reaction with any oxidizable species. One such oxidizable species is oxalic acid, which is used in fairly high concentration to precipitate and collect plutonium at the end of the purification process. Another oxidizable substance is stainless steel. All of the elements in stainless steel, including elemental chromium, will be oxidized by chromium(+6) and go into solution as the corresponding soluble ion. The overall effect of the reaction with chromium $(+6)$ is to enhance the corrosion process. The final two components of this work were to determine the rate of conversion of chromium $(+6)$ back to $\operatorname{chromium}(+3)$ via the reactions with oxalic acid and stainless steel.

An overall scheme for the reactions involving chromium in its various oxidation states is provided in Figure 1. 
II. CORROSION OF STAINLESS STEEL IN NITRIC ACID/HYDROFLUORIC ACID MIXTURES

Background

The stainless steel corrosion process is shown schematically in Figure 2. At some location on the surface exposed to the acid solution, oxidation of the elements in the alloy occurs, and the atoms move into solution in the form of their corresponding ions, leaving a number of electrons behind in the metal. At another location on the surface, these excess electrons are removed by reduction of protons at the metal-solution interface to generate hydrogen gas. Since there is no external source or sink for the electrons, the two reactions must occur at exactly the same rate. Thus, characterizing either of the reactions gives information about the other.

As the name would suggest, stainless steel does not corrode at an appreciable rate. The technique of placing a coupon of the sample in an electrolyte and measuring weight loss after a given time period is impractical since a very long time would be required to obtain a measurable weight loss. Most modern corrosion studies are instead done by 
electrochemical means. An external power supply is attached to the sample, and a potential greater than the equilibrium potential is imposed to increase the reaction rate. A direct measure of the reaction rate is the current density, and since the current density is related exponentially to the applied potential, a potential change in the order of a few hundred millivolts can increase the reaction rate by several orders of magnitude. This increase results in a corresponding decrease in the length of time required for the corrosion measurement.

A standard electrochemical corrosion measurement technique is one in which a series of potentials greater than the equilibrium potential is applied to the sample, and the equilibrium current density is measured at each applied potential. A Tafel plot is then constructed by plotting the applied potential versus the logarithm of the current density.1,2 A typical example of a lafel plot is shown in Figure 3. The exchange current density is the intersection of the linear portions of the plot and is directly proportional to the corrosion rate. The lower branch of the plot, with applied potentials more negative than the equilibrium potential, represents the enhanced reduction of protons. This is a single, usually uncomplicated, reaction and is very reproducible. The upper branch, as can be seen in the figure, is much more complicated because the enhanced oxidation reaction leads to the formation of passive oxide 
layers on the surface of the metal and other chemical reactions. This portion of the curve is much more difficult to reproduce. However, the exchange current density can still be obtained from the lower branch alone by extrapolating the linear portion of the curve back to the equilibrium potential line. This was the procedure used in this study.

Experimental

All corrosion measurements were made using a threeelectrode system. The working electrode consisted of a 5/8 in.-diameter 316 stainless steel rod encased in a sheath of teflon such that only the cross section of the rod was exposed to the solution (Figure 4). The surface disc was polished with 10-micron polishing alumina on a felt cloth before each series of measurements. When deep pitting occurred, it was turned down on a lathe to produce a fresh surface. A standard double junction silver/silver chloride reference electrode using $10 \%$ potassium nitrate outer filling solution was used for the low temperature measurements. However, both the glass-body and plastic-body electrodes were attacked by the hydrofluoric acid/nitric acid mixtures at elevated temperatures. For these measurements, a separate stainless steel rod was used as the reference electrode. Once this rod reached equilibrium with 
the solution, which was dependent on its own rate of corrosion, its potential drifted only slightly with time, allowing the corrosion rate of the sample to be measured with little or no error. A platinum wire was used as the counter electrode in the three-electrode measurement system.

All measurements were made using a Princeton Applied Research Model 273 potentiostat/galvanostat. Rather than applying a fixed current and measuring the equilibrium potential, which is the traditional measurement procedure, a linearly increasing current ramp was applied. The rate at which the current ramp was applied was slow enough to allow the system to always be at equilibrium. This change in procedure generated smooth curves that were easily analyzed, decreased analysis time, and allowed for unattended operation for periods up to 30 minutes once a scan was initiated.

All measurements were carried out in a teflon beaker fitted with a teflon stopper to decrease vapor loss. The beaker was placed in a water bath on a hot plate for temperature adjustments. The solutions were agitated with a magnetic stir bar and continually purged with argon before and during the measurements.

Examples of all calculations used in this study aie presented in Reference 3 . 
Results

Corrosion measurement results for 316 stainless steel in nitric-hydrofluoric acid mixtures are shown in Figure 5. As expected, the corrosion rates increase with an increase in temperature and hydrofluoric acid concentration. They are fairly insensitive, however, to nitric acid concentration. The most likely explanation for these results is that nitric acid operating alone tends to form a protective oxide layer on the surface of the stainless steel that inhibits further dissolution of the metal. Hydrofluoric acid acts to dissolve this oxide layer and expose Eresh surface for further reaction.

The measured corrosion rates ranged from $0.01 \mathrm{~mm} / \mathrm{yr}$ for room temperature nitric acid with no $\mathrm{HF}$ up to 6-7 mm/yr for nitric acid at $80^{\circ} \mathrm{C}$ with $0.4 \mathrm{M} \mathrm{HF}$. These values are in excellent agreement with reported literature values of 0.3 $\mathrm{mm} / \mathrm{yr}$ in boiling concentrated nitric acid with no $\mathrm{HF}$ and $11.2 \mathrm{~mm} / \mathrm{yr}$ in $1.67 \mathrm{M}$ nitric acid and $0.5 \mathrm{M} \mathrm{HF}$ at $80^{\circ} \mathrm{C} .4$ 
The most extreme conditions used for the recovery of plutonium are leaching of various scrap matrices in concentrated nitric acid with $0.4 \mathrm{M}$ HF under reflux conditions, approximately $120^{\circ} \mathrm{C}$ at atmospheric pressure. Using an Arrhenius-type plot for the data obtained in this study, the estimated stainless steel corrosion rate under these conditions is $22 \mathrm{~mm} / \mathrm{yr}$. This corrosion rate yields a chromium dissolution rate of $3.4 \times 10^{-4}$ grams of chromium/hr per $\mathrm{cm}^{2}$ of surface area exposed to the solution.

Clearly the more surface area exposed to the solution, the more chromium that will dissolve from the stainless steel. One dissolution process at LANL uses a stainless steel can $71 / 4$ in. in diameter and 9 in. tall. The can is usually filled with 4 liters of solution. The total surface area in contact with the solution is $1,134 \mathrm{~cm}^{2}$. The rate of appearance of chromium in the solution under reflux is calculated to be approximately $100 \mathrm{ppm} / \mathrm{hr}$. For other dissolver designs the rate of appearance of chromium will be proportional to the stainless steel surface area exposed. For example, a dissolver consisting of a 6 -in. diameter glass column fitted with a stainless steel end plate will introduce chromium into solution at a much slower rate.

Following dissolution, the dissolver solutions are usually cooled to room temperature, where the corrosion rate decreases to less than $1 \mathrm{~mm} / \mathrm{yr}$. If this solution were stored 
in a 6-in. diameter stainless steel pencil tank, the chromium concentration would continue to increase at a rate of approximately $5 \mathrm{ppm} / \mathrm{hr}$ or $120 \mathrm{ppm} /$ day. In solutions containing no fluoride or where the fluoride is bound in the form of metal-fluoro complexes, the corrosion rate will decrease by about another order of magnitude.

III. CONVERSION OF CHROMIUM $(+3)$ TO CHROMIUM $(+6)$

Background

The formal potential of the chromium $(+6) / \operatorname{chromium}(+3)$ redox couple is fairly positive with a value of $+1.33 \mathrm{~V}$ versus the normal hydrogen electrode. Of the substances normally found in plutonium process streams the only one capable of oxidizing chromium(+3) to chromium(+6), either directly or indirectly, is nitric acid. However the oxidizing power of nitric acid is not fixed and increases with increasing temperature and concentration. Therefore it was necessary to further study this reaction to measure the rate of conversion to the higher oxidation state.

Experimental 
The experimental apparatus consisted of a 500-mL round bottom flask fitted with a condenser. Two hundred $\mathrm{mL}$ of a solution containing $3000 \mathrm{ppm}$ chromium(+3) and a fixed nitric acid concentration were placed in the flask and heated with a heating mantle until boiling, approximately $111^{\circ} \mathrm{C}$ at ambient pressure. The solution was allowed to reflux continually over a period of up to 21 hours with periodic removal of a $1-\mathrm{mL}$ sample aliquot. At the end of the experiment, the samples were analyzed spectrophotometrically using the absorbance at $440 \mathrm{~nm}$ to determine the chromium $(+6)$ content.

Results

Data for the conversion of chromium(+3) to chromium(+6) at three different nitric acid concentrations are shown graphically in Figure 6 . In $7.7 \mathrm{M}(1: 1)$ nitric acid, the rate of oxidation of chromium(+3) at $111^{\circ} \mathrm{C}$ is negligibie even after 21 hours. In $10 \mathrm{M}$ acid concentration, there appears to be a 4 to 5 hour induction period followed by an oxidation rate of approximately $9 \mathrm{ppm} / \mathrm{hr}$. In concentrated nitric acid, the induction period is negligible and the oxidation proceeds at a rate of approximately $55 \mathrm{ppm} / \mathrm{hr}$. 
IV. REDUCTION OF CHROMIUM (+6) TO CHROMIUM (+3) WITH STAINLESS STEEL

Background

In acid solution chromium $(+6)$ reacts with the principal components of stainless steel according to the following equations :

$\mathrm{Cr}^{6+}+\mathrm{Cr}^{0}-->2 \mathrm{Cr}^{3+}$

$\mathrm{Cr}^{6+}+\mathrm{Fe}^{0}-->\mathrm{Cr}^{6+}+\mathrm{Fe}^{3+}$

$2 \mathrm{Cr}^{6+}+3 \mathrm{Ni}^{0}-->2 \mathrm{Cr}^{3+}+3 \mathrm{Ni}^{2+}$

The net result is an enhancement of the stainless steel corrosion reaction with a corresponding reduction of chromium $(+6)$ to chromium $(+3)$. With regard to the presence of chromium (+6) in process solutions, it is important to know the rate of this reduction reaction relative to the rate of chromium $(+3)$ oxidation as discussed in the preceding section.

Experimental 
These studies were carried out by placing $4 \mathrm{~g}$ of stainless steel mesh in $200 \mathrm{~mL}$ of a solution containing 3000 ppm chromium(+6), 7.7 M nitric acid, and varying concentrations of $\mathrm{HF}$. The surface area of the stainless steel was estimated to be approximately $40 \mathrm{~cm}^{2}$. The solutions were contained in a teflon beaker fitted with a teflon stopper to minimize vapor loss, especially at elevated temperatures, and were stirred continuously by means of a magnetic stir bar. Each individual experiment was allowed to run from 6 to 9 hours with periodic sampling. The samples were analyzed spectrophotometrically after a 10:1 dilution.

Results

Experimental data for this set of experiments is shown in Figure 7. At room temperature there is a negligible reaction between chromium $(+6)$ and 316 stainless steel, independent of the HF concentration. However when the temperature is raised to $80^{\circ} \mathrm{C}$, the reduction of chromium $(+6)$ proceeds at a rate of about $250 \mathrm{ppm} / \mathrm{hr}$ with no $\mathrm{HF}$ present and at a rate of almost $1000 \mathrm{ppm} / \mathrm{hr}$ in $0.4 \mathrm{M} \mathrm{HF}$.

Discussion 
The disappearance of chromium $(+6)$ in this experiment can only be due to its reaction with stainless steel, since there are no other reducing agents present in solution. Also, as was shown in the previous section, oxidation of: chromium $(+3)$ to chromium $(+6)$ is not possible under these reaction conditions. A comparison of the corrosion rates measured in section II of this report in the absence of chromium $(+6)$ to those of this section indicate that the presence of $3000 \mathrm{ppm}$ chromium $(+6)$ accelerates the rate of corrosion by a factor of approximately 75 with no HF added and by a factor of approximately 16 with $0.4 \mathrm{M}$ HF present at $80^{\circ} \mathrm{C}$.

V. REDUCTION OF CHROMIUM (+6) TO CHROMIUM (+3) WITH OXALIC ACID

Background

Chromium (+6) is a frequently used reagent in organic synthesis to bring about the oxidation of selected compounds. 5,6 Many studies have been done on determining the kinetics and mechanisms of these reactions. In plutonium aqueous recovery operations the organic compound most likely to be present is oxalic acid, which is used as a precipitating agent to separate the purified plutonium from 
the process solution. Oxalic acid can be oxidized by chromium $(+6)$ according to the following equation:

$$
\mathrm{H}_{2} \mathrm{Cr}_{2} \mathrm{O}_{7}+3 \mathrm{H}_{2} \mathrm{C}_{2} \mathrm{O}_{4}+6 \mathrm{H}^{+}->2 \mathrm{Cr}^{3+}+6 \mathrm{CO}_{2}+7 \mathrm{H}_{2} \mathrm{O}
$$

Normally the reaction kinetics would be determined by mixing the two reactants and measuring the rate of disappearance of chromium $(+6)$ by monitoring the absorbance at its absorbance maximum wavelength at $440 \mathrm{~nm}$. Unfortunately, a reaction intermediate also absorbs at this wavelength making this procedure unusable. It is possible however to track the build up of chromium $(+3)$, a reaction product, by monitoring its absorbance at $560 \mathrm{~nm}$. This wavelength represents an absorbance maximum for chromium $(+3)$ in a spectral region relatively free from interference by the reactants and reaction intermediates.

By monitoring only the final reaction product, detailed information about the mechanism of the reaction and the kinetics of the rate-determining step cannot be obtained However, information about the overall reaction is available and is sufficient for the purposes of this study.

The rate of appearance of chromium $(+3)$ is given by: $\mathrm{d}\left[\mathrm{Cr}^{3+}\right] / \mathrm{dt}=\mathrm{k}\left[\mathrm{Cr}^{6+}\right]^{\mathrm{x}}\left[\mathrm{H}_{2} \mathrm{C}_{2} \mathrm{O}_{4}\right] \mathrm{Y}$, where $\mathrm{k}$ is the reaction rate constant and $\mathrm{x}$ and $\mathrm{y}$ represent the overall reaction order for chromium $(+6)$ and oxalic acid respectively. By using a 
large molar excess of oxalic acid, the reaction can be made pseudo $x$ order in chromium $(+6)$, i.e. $d\left[\mathrm{Cr}^{3+}\right] / d t=k^{\prime}\left[\mathrm{Cr}^{6+}\right]^{x}$,

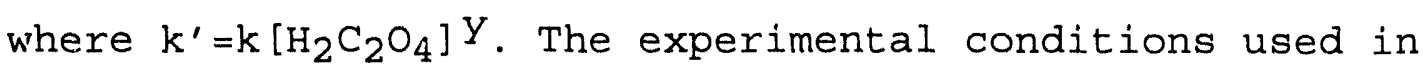
this study are listed in Table 1 . In each case the oxalic acid concentration is much larger than the chromium $(+6)$ concentration.

Experimental

The following three stock solutions were prepared: potassium dichromate in $7.7 \mathrm{M}$ nitric acid containing 1800 ppr chromium(+6), $50.0 \mathrm{~g} / \mathrm{L}$ oxalic acid in $7.7 \mathrm{M}$ nitric acid, and a solution containing only $7.7 \mathrm{M}$ nitric acid. These solutions were mixed together in various volume ratios giving differing concentrations of chromium $(+6)$ and oxalic acid while maintaining a constant concentration of nitric acid. The solutions were added directly to a standard 1-cm cuvette fitted with a stopper, shaken vigorously for approximately 10 seconds, and immediately placed in the spectrophotometer. Absorbance at $560 \mathrm{~nm}$ was measured as a function of time. All measurements were made on a HewlettPackard model 8452 UV-vis spectrometer.

Since there is some absorbance due to chromium $(+6)$ at $560 \mathrm{~nm}$, its contribution to the total absorbance had to be taken into account to accurately calculate the chromium $(+3)$ 
concentration in solution. The mathematical derivation for this procedure is presented in appendix $A$.

Results

An analysis of the kinetic data shows the rate of appearance of chromium(+3) to be first order in chromium $(+6)$. The standard first-order plot of log absorbance function versus time for all the experimental data is shown in Figure 8 . The slopes of the lines in the plot are equal to $-\mathrm{k}^{\prime} / 2.303$, which allows the calculation of the pseudo first-order rate constant. The results of these calculations are also presented in Table 1 . Since $\mathrm{k}^{\prime}=\mathrm{k}\left[\mathrm{H}_{2} \mathrm{C}_{2} \mathrm{O}_{4}\right]^{Y}$, a plot of $\log \mathrm{k}^{\prime}$ versus the $\log$ of the oxalic acid concentration should give a straight line whose slope is equal to $y$ and whose intercept is equal to $\log k$. This plot is shown in figure 9. The slope and intercept yield values of 1.74 and -5.241 respectively. Thus the overall reaction rate is given by: $d\left[\mathrm{Cr}^{3+}\right] / d t=5.74 * 10^{-}$ ${ }^{6}\left[\mathrm{Cr}^{6+}\right]^{1}\left[\mathrm{H}_{2} \mathrm{C}_{2} \mathrm{O}_{4}\right]^{1.74}$. Given a reaction stoichiometry of chromium $(+6)$ to oxalic acid of $1: 1.5$, the values for the exponents in the above equation are quite reasonable.

A similar study carried out in $2.43 \mathrm{M}$ perchloric acid showed the reaction to be first order in chromium $(+6)$ but 1st to 2 nd order in oxalic acid, depending on its concentration and the solution acidity. 7 
Discussion

One gram of oxalic acid per liter of solution is capable of reducing $385 \mathrm{ppm}$ of $\operatorname{chromium}(+6)$ to $\operatorname{chromium}(+3)$. Under the conditions used in this study and using the rate parameters derived above, it would take an estimated 4 days for the chromium (+6) concentration to reach less than 5 ppm. Doubling the oxalic acid concentration to $2 \mathrm{~g} / \mathrm{L}$ would decrease the reaction time to approximately 1 day. Also since chemical reactions tend to increase exponentially with an increase in temperature, the reaction vill take considerably less time at elevated temperatures. For example, passing a solution containing chromium $(+6)$ and oxalic acid through an evaporator as a final salt concentration step will cause the reduction of chromium(+6) to chromium $(+3)$ to occur at a much faster rate.

Conclusions

This study has investigated the generation and speciation of chromium in aqueous nitrate solutions as a function of nitric and hydrofluoric acid concentrations, temperature, and in the presence or absence of 316 stainless steel and oxalic acid. In Table 2 are listed some of the unit processes used in plutonium recovery operations along with the pertinent operating conditions as defined by these 
parameters. Each of these processes are discussed in turn as to the disposition of chromium in the resulting solutions.

Cascade Dissolver -6 in. Under the reaction conditions listed, an appreciable corrosion of the stainless steel end plates and heat exchanger tube will occur to produce a substantial chromium(+3) concentration in solution. Further oxidation to chromium $(+6)$ should occur at a rate of approximately $10 \mathrm{ppm} / \mathrm{hr}$. after a several hour induction period. However, the rate at wnich chromium $(+6)$ is reduced back to chromium $(+3)$ by the stainless steel will be greater than $1000 \mathrm{ppm} / \mathrm{hr}$. Therefore, even though there will be a substantial quantity of chromium in the solution, it will all be in the +3 oxidation state.

Cascade Dissolver -4 in. Due to the lower HF concentration the corrosion rate of the stainless steel components of the $4 \mathrm{in}$. dissolver will be lower than the rate in the 6 in. dissolver. The chromium concentration will still be appreciable but, for the same reasons stated above, there will be no chromium in the +6 oxidation state.

SS Pot Dissolver. Under these reaction conditions, there should be appreciable corrosion to the stainless steel container to give a solution high in $\operatorname{chromium}(+3)$. However the nitric acid concentration is too low to promote oxidation to chromium $(+6)$, even at elevated temperatures. 
Glass Pot Dissolver. Since there are no stainless steel components present in the apparatus, any chromium in solution has to have originated in the feed itself. If this is the case, then the reaction conditions are such that most or all of the chromium will be present in the +6 oxidation state, depending on how long the reaction is allowed to proceed.

Ion Exchange. The ion exchange feed is chemically treated to adjust plutonium to the +4 oxidation state. The rest potential of the solution then is in the region where chromium $(+3)$ is the thermodynamically stable oxidation state. Under the conditions of $7 \mathrm{M}$ nitric acid, very low free-HF concentration, and ambient temperature, the corrosion rate of stainless steel will be very low and all chemical reactions involving chromium, both oxidation and reduction, will be equally slow. Therefore, all chromium will be in the +3 state.

oxalate precipitation. In $1 \mathrm{M}$ nitric acid with no $\mathrm{HF}$ present, the corrosion rate of stainless steel will be very slow, and there should be no chromium in the original solution since this follows the ion exchange purification step. If chromium were present, the conditions would be too mild to oxidize it to the +6 state. And even if there were chromium $(+6)$ in the original solution, it would be reduced 
back to the +3 state by the reaction with excess oxalic acid.

Evaporator. In $4 \mathrm{M}$ nitric and low free-HF concentrations, oxidation of any chromium present to the +6 state will be very slow. However, the reactions of chromium $(+6)$ with stainless steel and oxalic acid to produce chromium(+3) will be fairly rapid by comparison.

Summary

According to the measurements made in this study, the only situation in which chromium $(+6)$ could exist in a plutonium process solution is one in which a feed containing chromium is dissolved in a glass pot dissolver in high nitric acid concentration and at high temperature. But when the resulting feed is prepared for ion exchange, the chemical treatment reduces chromium to the +3 state. Any solution being processed through the evaporator will only contain chromium in the +3 state and any chromium salts remaining in the evaporator bottoms will be chromium +3 salts. 
References

1. S. Evans and E. L. Koehler, "Use of Polarization Methods in the Determination of the Rate of Corrosion of Aluminum Alloys in Anaerobic Media," J. Electrochem. SoC., 108, 509 (1961).

2. "Standard Practice for Conventions Applicable to Electrochemical Measurements in Corrosion Testing." ASTM Designation: G 3-89.

3. "Standard Practice for Calculation of Corrosion Rates and Related Information from Electrochemical Measurements." ASTM Designation: G 102-89.

4. Corrosion Resistant Materials Handbook-4th ed. (Noyes Data Corporation, Park Ridge, NJ, 1985).

5. G. Cainelli, Chromium Oxidations in Organic Chemistry (Springer-Verlag, Berlin, 1984).

6. Oxidation in Organic Chemistry-Part A. K. Wiberg, Ed., (Academic Press, New York, NY 1965).

7. Fariza Hasan and Jan Rocek, "Three-Electron Oxidations. II. The Chromium(VI) Oxidation of Oxalic Acid," J. Amer. Chem. Soc., 94, 9073-9081, (1972). 
Appendix A. Kinetic Analysis for the Reaction of Chromium $(+6)$ with Oxalic Acid

The rate of conversion of chromium $(+6)$ to $\operatorname{chromium}(+3)$ was determined by measuring the absorbance at $560 \mathrm{~nm}$, an absorbance wavelength maximum for chromium $(+3)$. However, since chromium $(+6)$ also absorbs slightly at this wavelength, it was necessary to break down the total absorbance into the contribution from each of the two absorbing species. The mathematical analysis is as follows:

1) $\mathrm{Abs}_{t}=\mathrm{Abs}_{\mathrm{Cr}}(6+)+\mathrm{Abs}_{\mathrm{Cr}}(3+)$

$\mathrm{Abs}_{\mathrm{t}}=$ total absorbance at time $t$

Abscr $(6+)$ = absorbance due to $\mathrm{Cr}(6+)$

$\mathrm{Abs}_{\mathrm{Cr}}(3+)=$ absorbance due to $\operatorname{Cr}(3+)$

2) $\mathrm{Abs}_{t}=\left(\varepsilon_{\mathrm{Cr}}(6+) \times \mathrm{b} \times\left[\mathrm{Cr}^{6+}\right]_{t}\right)+\left(\varepsilon_{\mathrm{Cr}}(3+) \times \mathrm{b} \times\left[\mathrm{Cr}^{3+}\right]_{t}\right)$

$$
\begin{aligned}
& \varepsilon_{\mathrm{Cr}}(6+)=\text { the molar extinction coefficient for } \mathrm{Cr}(6+) \\
& \varepsilon_{\mathrm{Cr}}(3+)=\text { the molar extinction coefficient for } \mathrm{Cr}(3+) \\
& \mathrm{b}=\text { the cell path length } \\
& {\left[\mathrm{Cr}^{3+}\right]_{t}=\mathrm{Cr}^{3+} \text { concentration at time } t} \\
& {\left[\mathrm{Cr}^{6+}\right] t=\mathrm{Cr} 6+\text { concentration at time } t}
\end{aligned}
$$

3) $\left[\mathrm{Cr}^{3+}\right]_{t}=\left[\mathrm{Cr}^{6+}\right]_{0}-\left[\mathrm{Cr}^{6+}\right]_{t}$

$$
\left[\mathrm{Cr}^{6+}\right]_{0}=\mathrm{Cr}^{6+} \text { concentration at time zero }
$$


4) $\mathrm{Abs}_{t}=\left(\varepsilon_{\mathrm{Cr}}(6+) \times \mathrm{b} \times\left[\mathrm{Cr}^{6+}\right]_{t}\right)+\left(\varepsilon_{\mathrm{Cr}}(3+) \times \mathrm{b} \times\left\{\left[\mathrm{Cr}^{6+}\right]_{0}\right.\right.$ $-[\mathrm{Cr} 6+] t\})$

5) $\left[\mathrm{Cr}^{6+}\right]_{t}=$

$$
A b s_{t}-\varepsilon_{\mathrm{Cr}}(3+) \times \mathrm{b} \times\left[\mathrm{Cr}^{6+}\right]_{0}
$$$$
\left(\varepsilon_{C r}(6+) \text { x b }\right)-\left(\varepsilon_{C r}(3+) \quad \text { x b }\right)
$$

$$
\text { Abst }-\left\{\mathrm{Abs}_{\mathrm{n}} /\left[\mathrm{Cr}^{3+}\right]_{\mathrm{n}}\right\} \times\left[\mathrm{Cr}^{6+}\right]_{0}
$$

6) $\left[\mathrm{Cr}^{6+}\right]_{\mathrm{t}}=$

$$
\left(\mathrm{Abs}_{\mathrm{O}} /[\mathrm{Cr} 6+]_{0}\right)-\left(\mathrm{Abs}_{\mathrm{n}} /\left[\mathrm{Cr}^{3+}\right]_{\mathrm{n}}\right)
$$

$\left[\mathrm{Cr}^{3+}\right]_{\mathrm{n}}=\mathrm{Cr}^{3+}$ concentration at the end of the reaction $\mathrm{Abs}_{\mathrm{O}}=$ the initial absorbance value $\mathrm{Abs}_{\mathrm{n}}=$ the final absorbance value

7) $\left[\mathrm{Cr}^{3+}\right]_{\mathrm{n}}=\left[\mathrm{Cr}^{6+}\right]_{0}$

$$
A b s_{t}-\left(\mathrm{Abs}_{\mathrm{n}} /\left[\mathrm{Cr}^{6+}\right]_{0}\right) \times\left[\mathrm{Cr}^{6+}\right]_{0}
$$

8) $\left[\mathrm{Cr}^{6+}\right]_{t}=\ldots \ldots$

$$
\left(\mathrm{Abs}_{0} /\left[\mathrm{Cr}^{6+}\right]_{0}\right)-\left(\mathrm{Abs}_{\mathrm{n}} /\left[\mathrm{Cr}^{6+}\right]_{0}\right)
$$

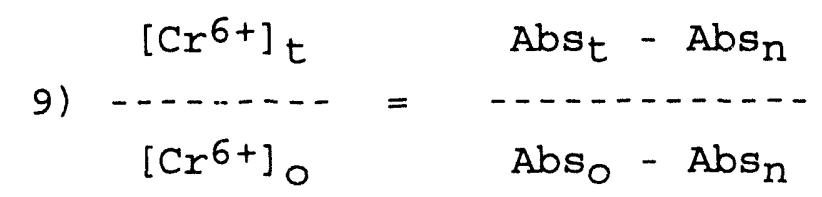

For a reaction pseudo first-order in $\operatorname{chromium}(+6)$, the rate equation is given by: $-d\left[\mathrm{Cr}^{6+}\right] / d t=k^{\prime}\left[\mathrm{Cr}^{6+}\right]$. The solution to this equation is: 
10) $\log \left(\left[\mathrm{Cr}^{6+}\right]_{t} /\left[\mathrm{Cr}^{6+}\right]_{0}\right)=-\mathrm{k}^{\prime} t / 2.303$.

Substituting Equation 9) into Equation 10) yields:

11) $\log$\begin{tabular}{cc}
$A b s_{t}-A b s_{n}$ & $-k \cdot t$ \\
\hline$A b s_{0}-A b s_{n}$ & 2.303
\end{tabular}

Therefore a plot of the log of this absorbance function versus time should give a straight line whose slope is equal to $-k^{\prime} / 2.303$

A similar absorbance function was derived for a reaction pseudo second-order in $\operatorname{chromium}(+6)$. However the experimental data did not fit the second-order plot so the derivation is not provided here. 


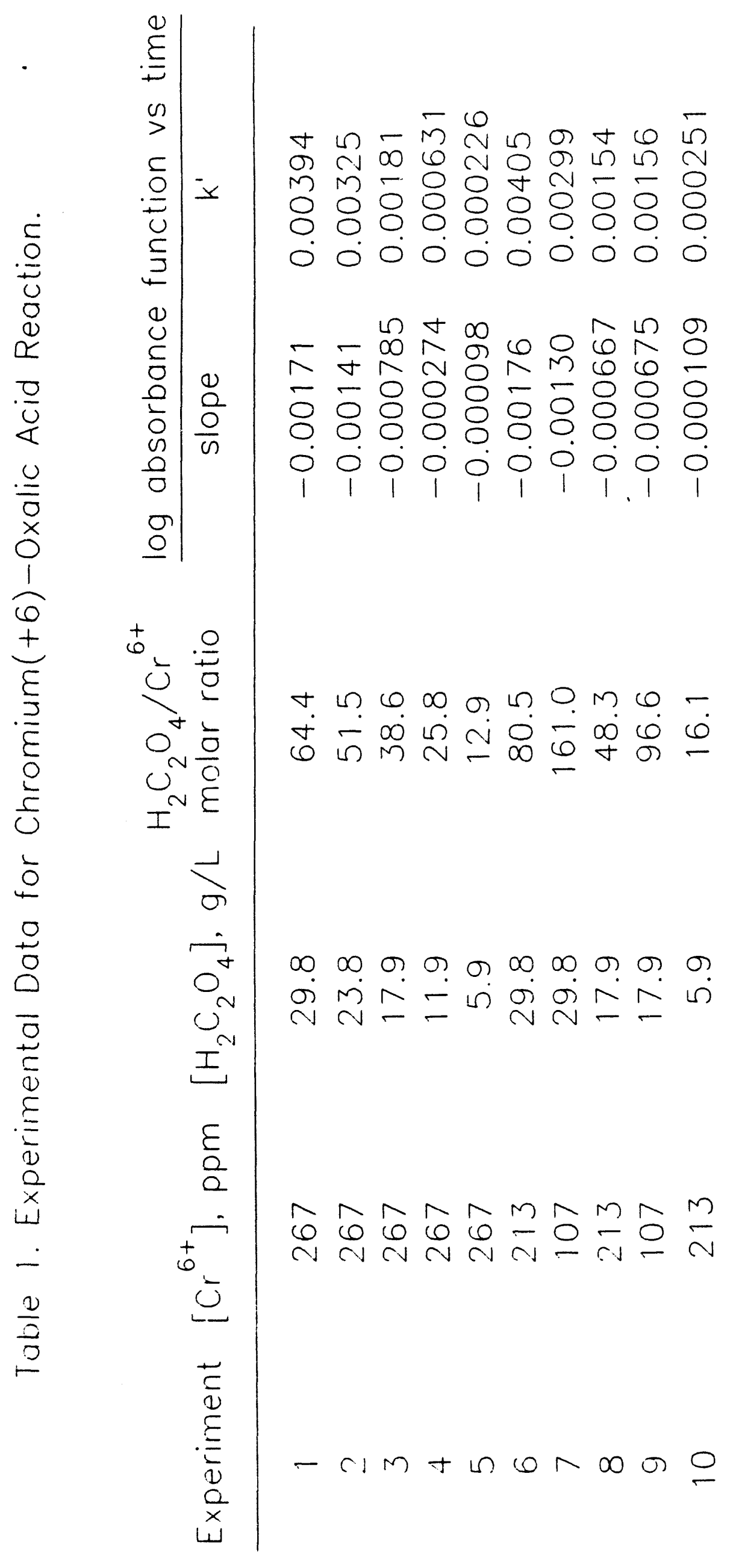


Table 2. Operating Parameters for Plutonium Recovery Operations Unit Processes.

\begin{tabular}{|c|c|c|c|c|c|}
\hline Process & {$\left[\mathrm{HNO}_{3}\right], \mathrm{M}$} & [HF], $M$ & Tomp, ${ }^{\circ} \mathrm{C}$ & SS present & Oxalic Acid, g/L \\
\hline Cascade Dissolver-6 in. & $8-10$ & $0.1-0.5$ & $90-105$ & end plate, heat tube & none \\
\hline Cascade Dissolver-4 in. & $10-15$ & $0.1-0.2$ & $90-105$ & ond plate, heat tube & none \\
\hline Ss Pot Diasolver & $4-8$ & $0.1-0.3$ & 114 & all & none \\
\hline Glass Pot Dissolver & $12-15$ & $0.1-1$ & 114 & none & none \\
\hline Ion Exchange & 7 & $<0.1$ & $20-30$ & all or end plate & none \\
\hline Oxalate Precipitation & 1 & 0 & $20-30$ & all & $9-18$ \\
\hline Evaporator & 4 & $<0.1$ & $110-114$ & all & none, 9-18 \\
\hline
\end{tabular}


Figure 1. Chromium Reactions in Plutonium Process Solutions

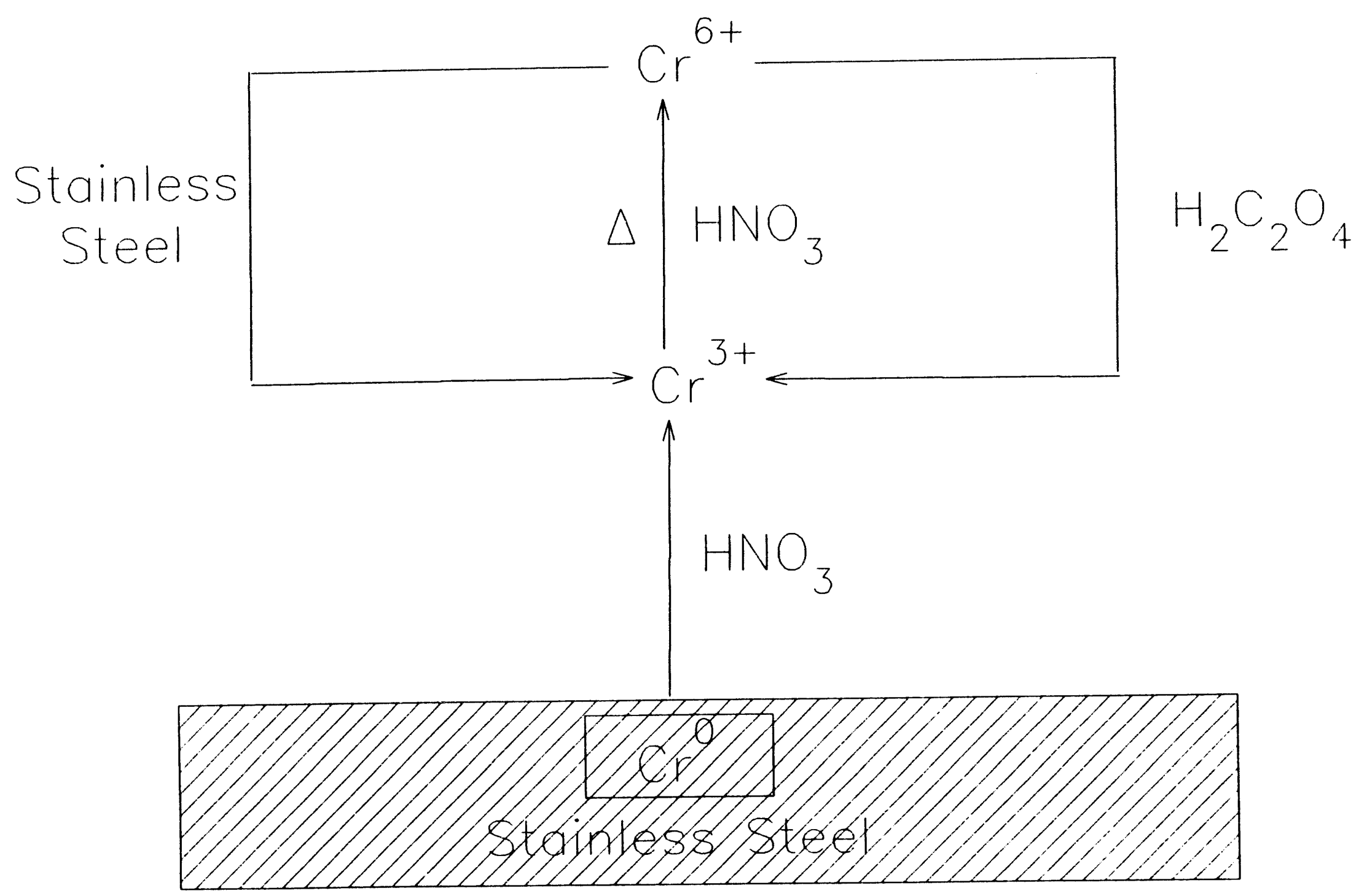




\section{Corrosion of Stainless Steel in Acid. Solution}

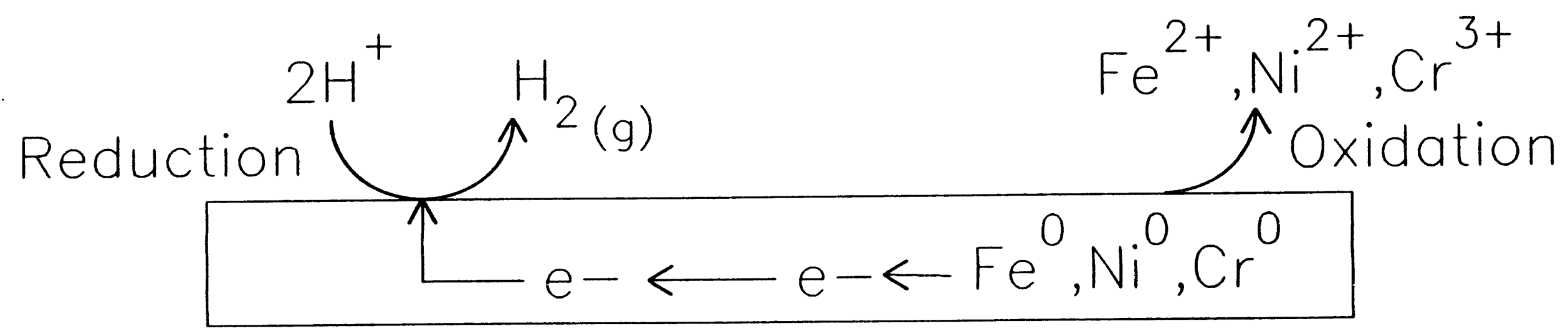

Stainless Steel 

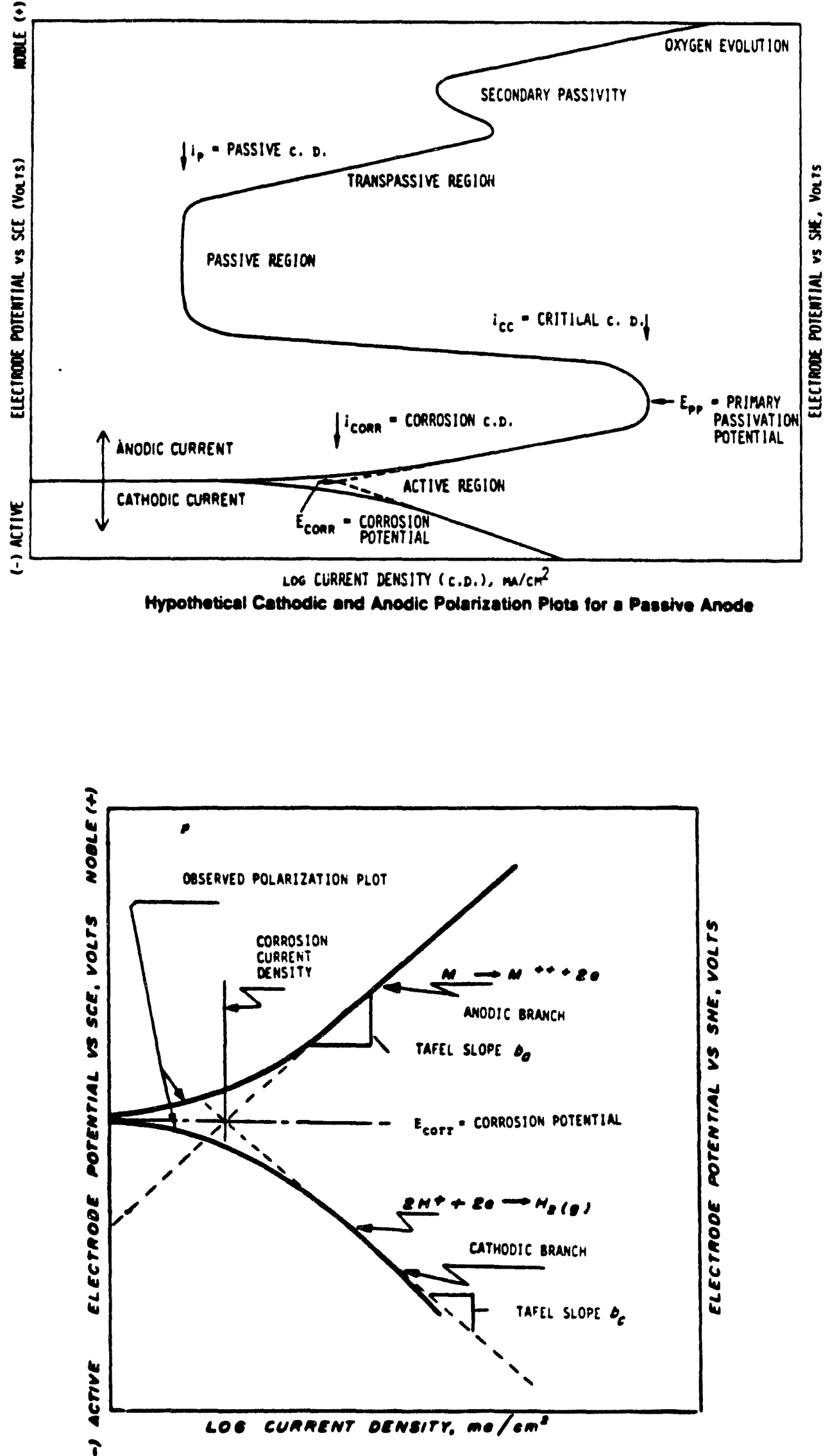
Figure 5. Corrosion Rates for 316 Stainless Steel
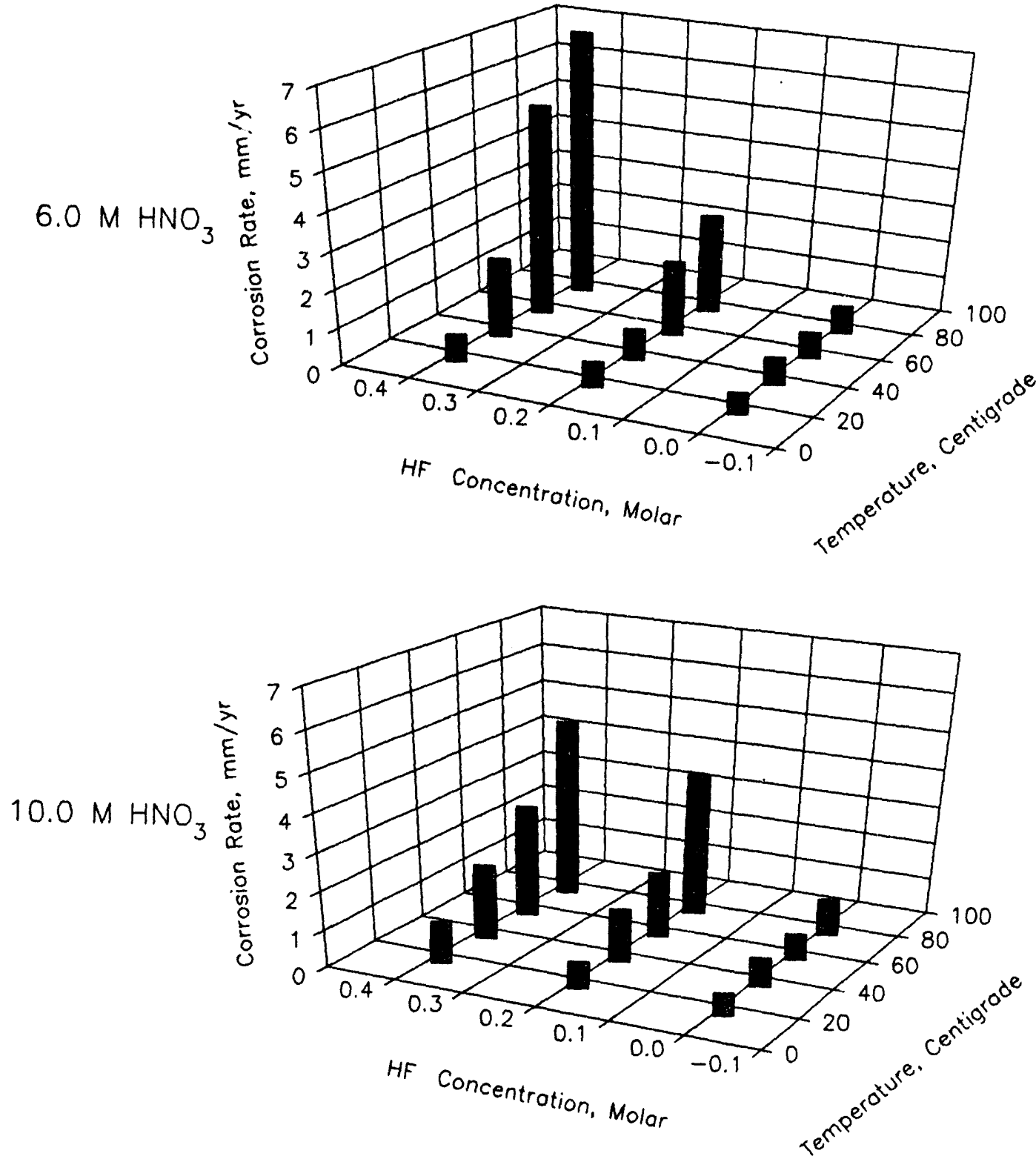

$15.6 \mathrm{M} \mathrm{HNO}_{3}$

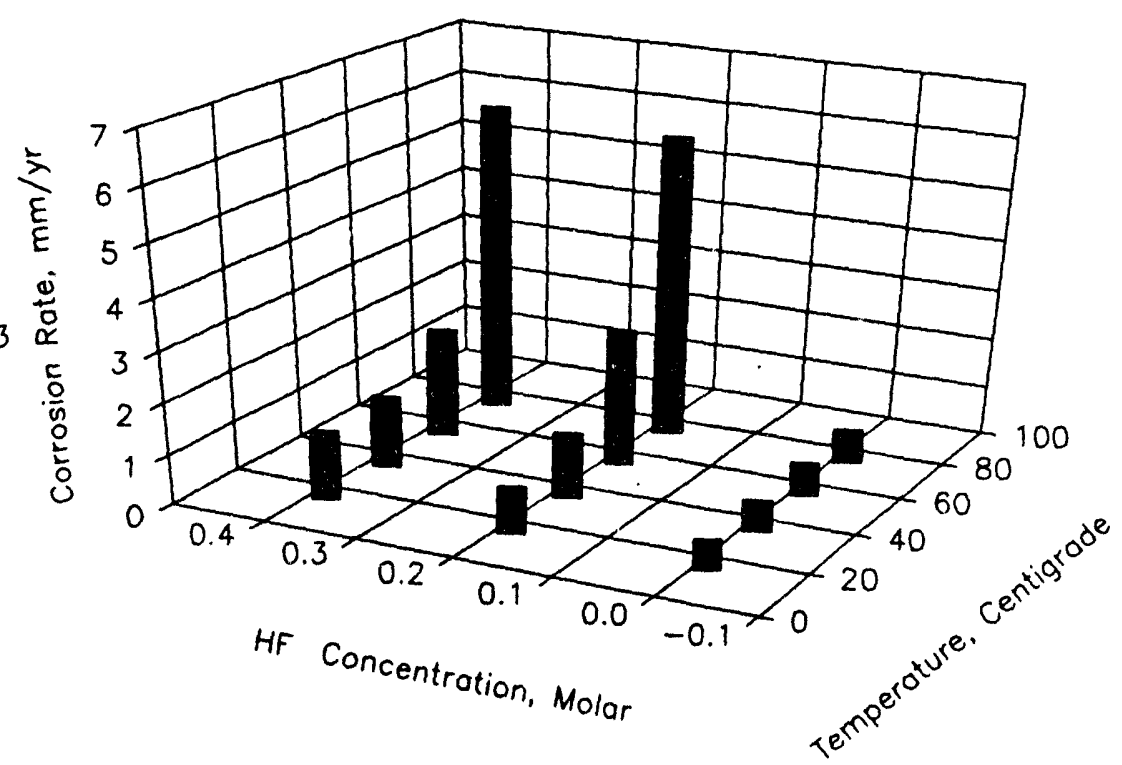


Figure 7. Reduction of Chromium(+6) With 316 Stainless Steel in 7.7 M Nitric Acid-Hydrofluoric Acid Mixtures

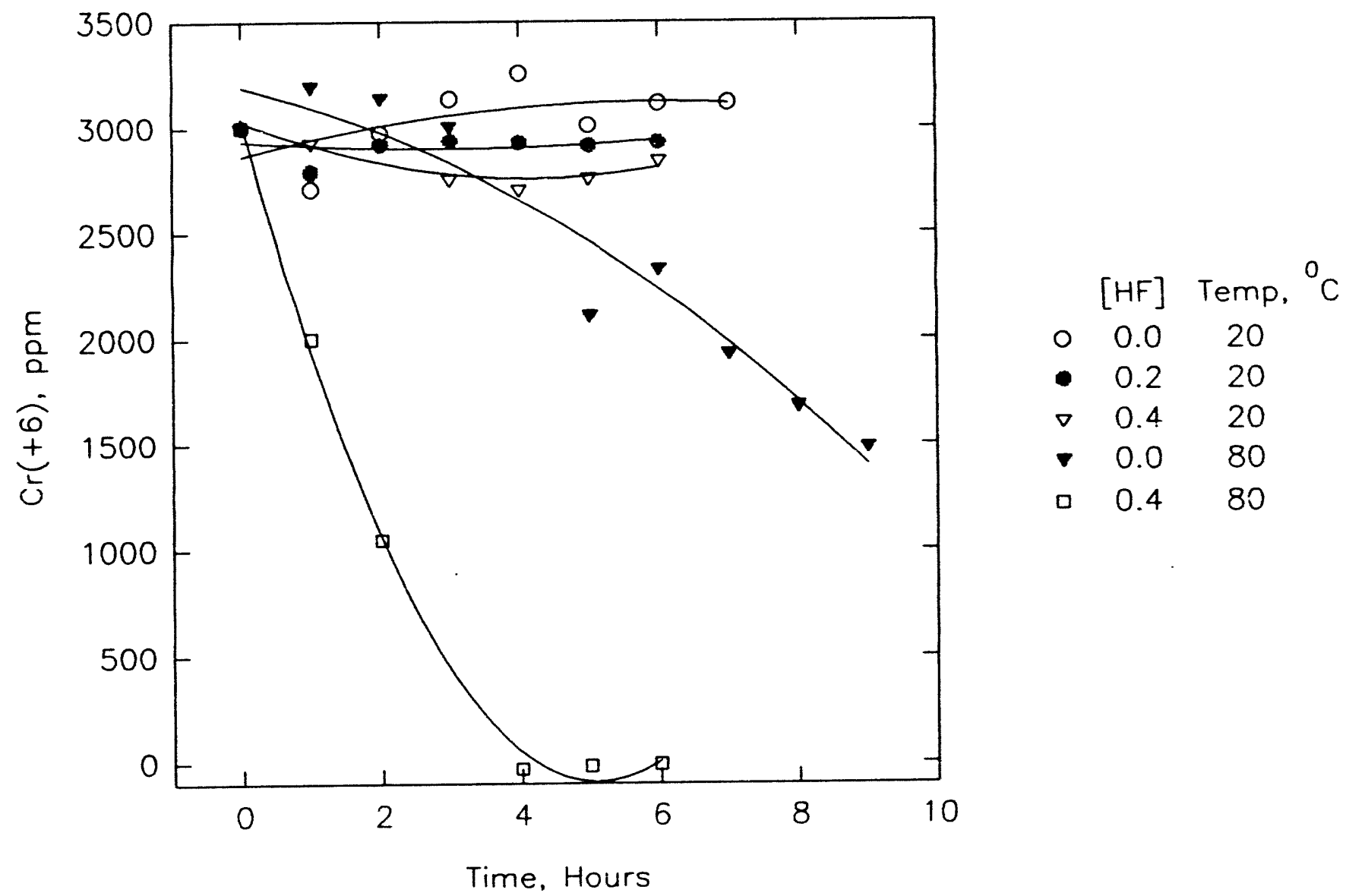


Figure 8

Chromium-Oxalic Acid Reaction

First Order Kinetic Analysis

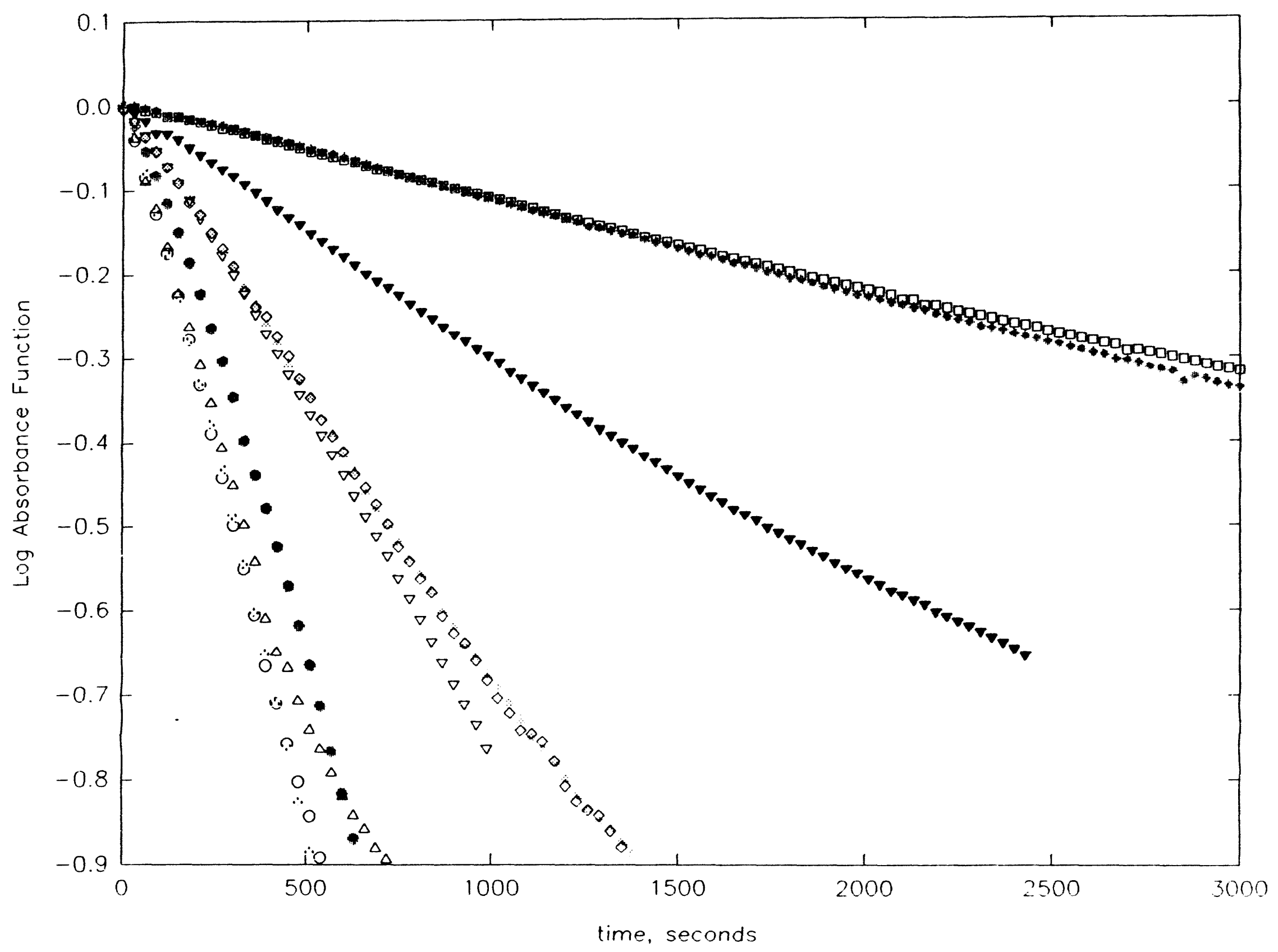



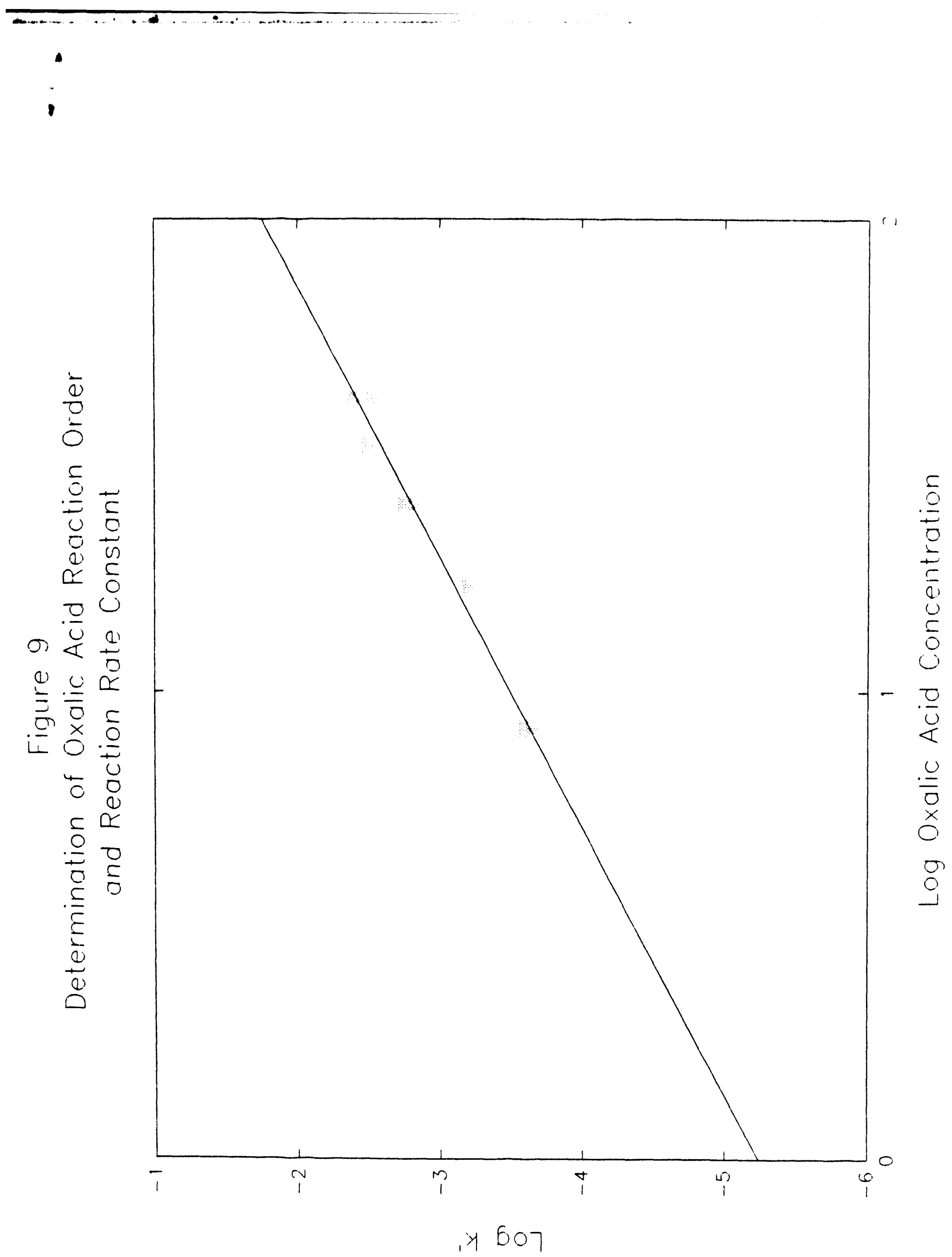

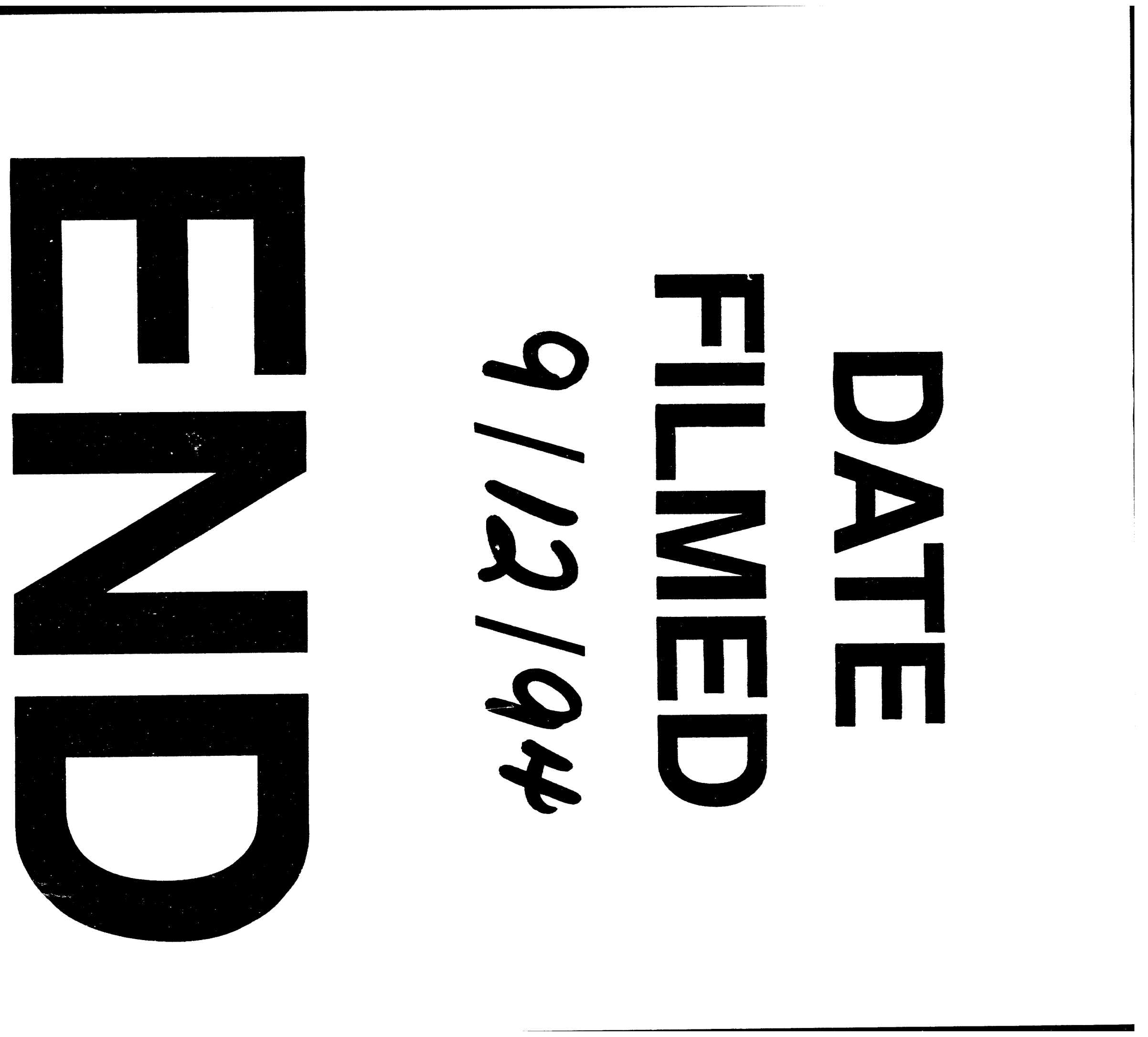


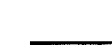
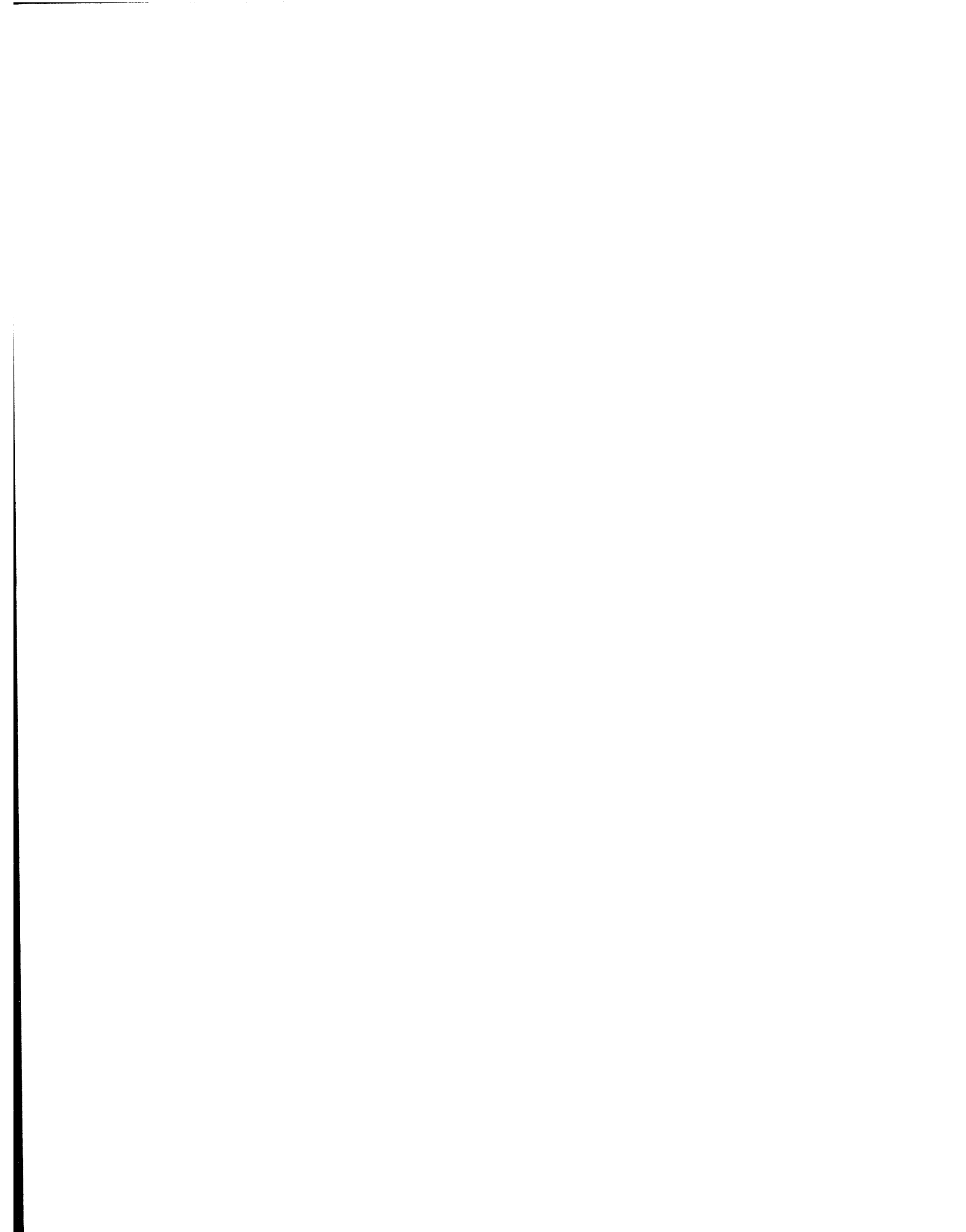